\title{
The peer-review process in medical publishing: A reviewer's perspective
}

Frank W. Sellke, MD

See related editorial on page 1681.
From Beth Israel Deaconess Medical Center, Harvard Medical School, Boston, Mass. Supported by HL 46716 and HL 69024.

Received for publication July 16, 2003; accepted for publication July 28, 2003.

Address for reprints: Frank W. Sellke, MD, Beth Israel Deaconess Medical Center, Harvard Medical School, 110 Francis St, LMOB 2a, Boston, MA 02215 (E-mail: fsellke@bidmc.harvard.edu).

J Thorac Cardiovasc Surg 2003;126:1683-5

Copyright () 2003 by The American Association for Thoracic Surgery

$0022-5223 / 2003 \$ 30.00+0$

doi:10.1016/j.jtcvs.2003.07.014

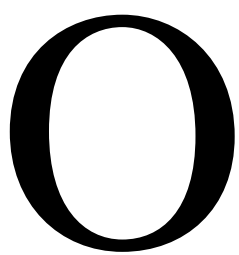

ne of the hallmarks of academic surgery, or medicine in general, is the dissemination of new information from personal experience, clinical trials, and laboratory investigation. Recently, this has taken several new forms with the advent of computer-based presentations and graphics and the use of Web-based media in addition to old, standard, hard-copy journals. Whatever the media format, to make certain that the new information has a reasonable likelihood of being truthful and clinically or scientifically relevant, peer review has been used as a judge of validity, importance, and quality of presentation. Since its inception, the peer-review process has been criticized by some as slowing this dissemination of knowledge. In fact, several Nobel Prize-winning findings have been rejected for publication during the initial review. However, without this screening process, most of what we read or hear would not be worth the time required to absorb it. The following will describe my personal view of the process as a frequent reviewer for several medical and scientific journals.

\section{Why Do Peer Review?}

The peer-review process generally involves having one's academic work judged by several peers, which by definition means equals, but in fact, the peers are usually persons with some perceived exceptional knowledge or experience in the subject area being evaluated. First and foremost, the peer-review process provides expert opinion regarding the quality and appropriateness of research. Are the findings important and relevant? This is the case whether findings are the result of a large clinical trial evaluating the use of drug-eluting stents and coronary artery bypass surgery, the use of a new molecular target for the treatment of childhood leukemia, or the discovery of a new nuclear particle. The study might involve a large number of patients being subjected to several hazardous treatments, have cost millions of dollars, and required 10,000 man-hours of work. However, if the findings are well established, there is no reason to publish them again. The methods used can be very diverse and might depend as much or more on what is available to the investigator (as least as far as laboratory investigation is concerned) as anything else. However, it is the reviewer's job to judge the appropriateness of the methods in determining how the results were generated. Another major area to be assessed is whether the results of the study justify the conclusions. Often, these might seem quite simple, such as "this study finds that aortic valve replacement can be performed in octogenarians with acceptable results." However, it is often not this straightforward, as in a basic science investigation of a new molecular pathway using novel laboratory methods. Although certainly taking a secondary role, a reviewer should assess whether the findings are interesting and well presented. Finally, the reviewer should determine whether there are any laboratory animal or patient welfare concerns.

If this is such a good process, why is it not perfect? Well, most of those who have submitted a paper for publication have received a rejection letter. Our first response is usually, "Those idiot reviewers didn't understand what I was doing and do not realize that this is the greatest work ever done!!" First of all, if an investigator does not feel that his work is of major importance, the work is probably not worth doing. However, the authors might be somewhat biased regarding the quality and effect of their research. This is not to say that the reviewers might also be biased against the 
authors and might not understand the potential clinical effect of the study, and some reviewers might not be adequately knowledgeable to assess whether the work is important and well executed. That is why there is an editor to act as the judge and 2 or more reviewers to provide the evidence for the final judgment. If 3 reviewers come to the same conclusion, they are usually correct in their assessment. When a young clinician or scientist is asked to review a paper, he or she might not have experience in the review process and might not have written enough articles to judge whether the information is well presented or if the methods are appropriate or optimal. This can also be said for busy, established surgeons and scientists who have many other things to do besides review papers. Finally, most of us know of the frustration of waiting several months to receive the review of a paper, when we always send our reviews to the editor within a week or 2 of its receipt. In reality, most surgeons would rather operate and play golf than spend their time reviewing papers. Often they rationalize doing them as a last resort or only after the editor has sent several nasty notices of the delinquent review. Furthermore, once a relatively favorable review is obtained, the editor usually requires revisions before the paper is finally accepted. This can require that the paper be sent out again to the same set of slow reviewers. Thus the process can move very slowly and take many months before the paper is accepted, if it ever is. Finally, there is usually a considerable lag between the time of the acceptance and the ultimate publication of the article in the journal.

Despite these shortcomings, the peer-review process is a good method to screen research for publication. It is the responsibility of the editor to make certain that the reviews are fair, unbiased, and accurate. One reviewer's or editor's opinion might not be a universal indicator of the quality or importance of research. However, just as a democracy might not be a good form of government but simply the best one around, the peer-review process is the best we have.

\section{What Is a Good Paper?}

Although the quality of a study or the paper describing it is very subjective, the editor usually outlines how he would like the reviewer to make an assessment, as is the case for the Journal of Thoracic and Cardiovascular Surgery. In general, a good paper describes a novel study that tests a hypothesis rather than simply presenting a series of clinical cases or relatively boring laboratory research findings. Even clinical research should test a hypothesis, and this should be clearly described in the first part of the paper. The abstract is usually the only portion of the paper that is read, and therefore it is critical that it briefly yet accurately describes the methods, results, and conclusions of the study. The findings should be important and clinically or scientifically relevant. The methods and statistical analysis should be appropriate and adequately rigorous. As mentioned above, the conclusions should follow from the results. The paper should present honest work that has not been fabricated or inappropriately altered. Although the latter statement should go without saying, not infrequently are data submitted that appear improbable.

Assuming that the methods and findings are adequate, the presentation is also important. If someone cannot get the meaning from a journal article, why should it be published? In general, the text, figures, and tables should present the data clearly and in a manner that is to the point without any duplication of the same information in the text, figures, or tables. Also, the figure legends should give enough information so that someone can interpret the information found in the figures, but they should not repeat a description of the data. The "Discussion" section should briefly summarize the important findings and explain the importance and context of the results, along with the limitations of the methods and results. This can, in general, be easily accomplished within 4 typed, double-spaced pages. The references should be appropriate but not excessive (usually no more than 25-35).

In a less-than-optimal paper, there is no hypothesis or the paper is purely descriptive. Although some important studies do present solely descriptive information, these should be limited to those initially describing a novel finding. Other factors influencing the merit of a paper are fatally flawed methods, incorrect statistical analysis, or a reexamination of information presented in studies already published. If the results do not support the conclusions, the paper is almost universally rejected. A verbose or poorly organized style lessens the enthusiasm of most reviewers. If critical references are omitted or if an author only references his or her own articles, the paper is judged more poorly. Always check the referenced authors for membership on the editorial board of the journal to which you are submitting the paper. Chances are that this person will review the paper. Little turns off a reviewer more than having his or her name repeatedly misspelled in the references. Although some studies, especially those dealing with translational research, do mandate many authors, most clinical reports having more authors than subjects requires some explanation.

\section{What Makes a Good Reviewer?}

A good reviewer, almost by definition, gives an unbiased and knowledgeable assessment of the quality and presentation of clinical or laboratory research. Importantly, he or she supports the statements with references or an analysis of the information presented. If the reviewer claims that the paper describes "nothing new," he should give some references to support the statement. The reviewer should always make his statements in a constructive manner and refrain from making inflammatory comments, even when he or she feels they 
are deserved. In fairness to the authors and editor, the reviewer should finish his assessment within 2 to 3 weeks. He has to do it eventually, so he might as well get it over with.

\section{Ethical Issues}

The reviewers and editors unfortunately on occasion need to address ethical issues. Fabricated data can be difficult if not impossible to detect, unless the same fabricated data is published in several articles or if the behavior is of an egregious nature or monumental scale. This has occurred more than once in even the most respected journals and in the laboratories of some very well-known investigators. The submission of identical or nearly identical data in different papers is not acceptable unless they are gathered from different experiments and are used to support the conclusions in another study, or it is acknowledged that some data are repetitive of that published in another article. Duplicate or simultaneous publication or submission of identical papers is also not allowed. It is also a dangerous practice because the same reviewers often receive papers from different journal editors. It is only a matter of time before the same reviewer receives the same paper from 2 different editors at the same time. Also, knowingly failing to acknowledge prior published work of another author or knowingly failing to reference the author's similar published findings is not acceptable. However, if an author simply is not aware of similar work published in his research area, this might not necessarily be unethical. It might only be poor research technique caused by a failure to read journal articles.

Justification of authorship has received considerable attention recently but remains subjective. One occasionally used criterion is a senior authorship for someone who has no idea of what is happening in the laboratory, but he or she has been the leader for so long that everyone is afraid to not include him on every paper. This actually lessens the credit received by those who actually did the work and wrote the paper. A second rather subjective criterion is being in the same laboratory area around the same time as when the work was performed. Although credit should be given for work performed, this has limits. On rare occasions, authorship is given for sexual or other inappropriate tasks having nothing to do with research. Lack of informed consent and misuse of laboratory animals is now relatively rare, but it is the duty of the reviewer to determine whether there is evidence that it has occurred or that good research practice has not been maintained.

Unethical behavior is not limited to the authors. A reviewer rejecting or giving a scathing review on a competitor's paper is not ethical. Also, rejecting an author's paper when the findings contradict the published results of the reviewer is not ethical behavior. When a paper is received that is authored by a friend or colleague, it is not acceptable to give preferential treatment or accept it simply because the author is your friend. It is better for the reviewer to disqualify himself or herself. Finally, accepting a paper on the basis of how many times the author has referenced the reviewer's papers is not good practice.

In summary, the peer-review process might have some shortcomings, such as potential bias of reviewers, honest difference of opinion that might translate into a rejected paper, and frequent slow pace of processing the paper from submission until it is published. Despite these limitations, peer review is probably the best method to ensure a timely publication of new, highly significant, and honest medical or scientific information. 